\title{
Placebo controls in clinical trials: concerns about use in relapse prevention studies in schizophrenia
}

Robin Emsley and colleagues question the use of placebos when established treatment is effective and lack of harm has not been proved

\author{
Robin Emsley Sarah Turoff, endowed chair in schizophrenia research ${ }^{1}$, W Wolfgang Fleischhacker \\ professor $^{2}$, Silvana Galderisi professor ${ }^{3}$, Lisa J Halpern director of recovery services ${ }^{4}$, Joseph $\mathrm{P}$ \\ McEvoy professor ${ }^{5}$, Nina R Schooler professor ${ }^{6}$
}

\begin{abstract}
${ }^{1}$ Faculty of Medicine and Health Sciences, Stellenbosch University, PO Box 241, Tygerberg Campus 8000, Cape Town, South Africa; ${ }^{2}$ Department of Psychiatry and Psychotherapy, Medical University Innsbruck, Innsbruck, Austria; ${ }^{3}$ Department of Psychiatry, University of Naples, Naples, Italy; ${ }^{4}$ Vinfen, Cambridge, MA, USA; ${ }^{5}$ Department of Psychiatry and Health Behavior, Georgia Regents University, Augusta, Georgia, USA; ${ }^{6}$ State University of New York, Brooklyn, NY, USA
\end{abstract}

The use of placebos in clinical trials has major policy implications for ethical conduct across all of medicine and is relevant to clinicians, patients, drug development, and regulatory agencies. This article focuses on the use of placebos in relapse prevention studies in schizophrenia. However, the issues discussed are similar to those encountered in many other clinical trial situations. These include underestimating the risk of harm associated with trial participation, the risk of coercion, insufficient awareness of the risks by participants, and the risk of loss of trust between the patient and doctor.

While the debate around using placebos in clinical trials of schizophrenia is long running, several developments make it imperative to readdress the topic. Firstly, new research has reported deleterious effects of relapse, ${ }^{1}$ challenging the previous assumption that relapse is not associated with a risk of lasting harm. Secondly, new questions have been raised about the need for maintenance treatment in schizophrenia. ${ }^{2}$ Thirdly, ethical standards have evolved, with reduced tolerance of exposure of participants to risk and greater respect of patient autonomy. Finally, and most importantly, recent publications from both the European Medicines Agency and US Food and Drug Administration continue to encourage the use of placebos in schizophrenia trials.

\section{Maintenance medication for schizophrenia}

The place of maintenance antipsychotic treatment in schizophrenia has been questioned for two reasons. ${ }^{2}$ The drugs have potentially serious adverse effects, and preliminary and uncontrolled evidence suggests that in some patients gradual dose reduction and, where feasible, discontinuation may be associated with better long term outcome. ${ }^{3}$ However, stopping antipsychotic treatment is associated with very high rates of relapse, even after a single episode of psychosis. ${ }^{4}$ Relapses may be associated with undue patient suffering, detrimental effects on social and vocational function, delayed time to treatment response, emergent treatment refractoriness, and reduction in brain volume. ${ }^{1}$ Furthermore, predictors of relapse are unreliable, measures to identify early signs of relapse are not always effective, and rescue interventions may not always prevent recurrence of severe illness. ${ }^{5}$ These concerns, together with the fact that the effectiveness of maintenance antipsychotic medication is one of the best documented findings in psychiatry, ${ }^{6}$ indicate that the benefits of maintenance treatment clearly outweigh the risks.

The efficacy and safety of antipsychotics for maintenance treatment of schizophrenia have been established through clinical development programmes, of which the placebo controlled, randomised, controlled trial has been a key component. Given the high rates of relapse associated with placebo treatment, it is not surprising that concerns have been raised regarding the risks to participants exposed to placebo in randomised trials. ${ }^{78}$ The topic was discussed at a full symposium of 4th Schizophrenia International Research Society Conference in April 2014.

Unlike in clinical settings, where the decision to discontinue treatment is largely patient driven, in trials the decision to withhold active treatment is instigated by clinicians. This is in direct contrast to the usual focus of clinicians on promoting adherence to treatment. ${ }^{9}$ Risk to trial participants is likely to be greatest in trials of maintenance treatment or relapse prevention, where patients, once stabilised, are switched to placebo until sufficient relapse events have occurred to be able to show a treatment effect. Indeed, the absolute risk difference for antipsychotics versus placebo in relapse prevention studies is 
more than double that in acute trials. ${ }^{10}$ Despite these concerns, current practice permits the use of placebos in such settings and various stakeholders promote these trials.

\section{Ethical position}

The World Medical Association's Declaration of Helsinki regards the use of placebos as acceptable in studies where there is no proved intervention, or where compelling and scientifically sound methodological reasons exist for the use of placebos to determine efficacy or safety of an intervention, and where patients who receive placebos will not be exposed to any risk of serious or irreversible harm. The declaration states that extreme care should be taken to avoid abuse of this option, and that the interest of science and society should never take precedence over considerations related to the wellbeing of individual patients. ${ }^{11}$

The International Conference on Harmonisation guideline on choice of control group ${ }^{12}$ considers a placebo control to be generally inappropriate when an available treatment is known to prevent serious harm in the study population. The document specifies that there are occasional exceptions, such as when standard therapy has toxicity so severe that many patients have refused to receive it.

\section{What the regulatory authorities say}

Regulatory authorities in North America and the European Union continue to encourage placebo controls in relapse prevention studies for the licensing of new drugs. The EMA's recent guidelines for trials of new treatments for schizophrenia states that, to show that a treatment maintains effectiveness over time, the inclusion of a placebo arm is possible and appropriate in a randomised withdrawal study (the standard design in placebo controlled relapse prevention studies) as long as it is appropriately designed and conducted. ${ }^{13}$ The document emphasises that these studies need to be conducted in highly controlled settings, with appropriate safeguards. It states that in this setting the benefits of a placebo arm will generally over-ride ethical reservations and that there should not be ethical problems if patients who relapse receive immediate active treatment. $^{13}$

A draft guidance on enrichment strategies for clinical trials by the FDA describes the randomised withdrawal design as a way to establish long term effectiveness of drugs when protracted use of a placebo would not be acceptable. In this design the study population receives active treatment for an extended period and those who respond enter a blinded, randomised treatment withdrawal phase for a short duration. Patients are withdrawn from the study if their symptoms recur, thereby minimising exposure to placebo. ${ }^{14}$

\section{Arguments for continued use of placebo}

There is a moral imperative to guard against ineffectual treatments being approved for use in clinical practice. A comparison between the investigational drug and placebo is considered the most powerful method of establishing efficacy and its use in randomised trials in schizophrenia has been regarded as both ethically and scientifically justifiable when supported by sound methodological considerations and its use does not expose participants to excessive risks of harm. ${ }^{15}$ The ongoing inclusion of placebos in relapse prevention studies has been justified on the basis of there being no clear evidence of increased risk of persistent morbidity or mortality, and because alternative study designs may not be as good at demonstrating efficacy and tolerability. It has been argued that use of placebos can be considered to be safe and ethical based on three premises:

- Available empirical evidence indicates no increased risk of severe harm or long term morbidity after exposure to placebos

- The belief that clinical measures can be put in place to effectively detect early symptoms and prevent serious relapse

- The likelihood that from a statistical perspective fewer relapses would be necessary to detect a positive outcome with placebos rather than with an active comparator $^{16}$

\section{Our concerns}

We believe that all three of these assumptions may be flawed. Relapses can cause lasting harm, and "rescue" interventions in trials are not always effective in averting relapse. Furthermore, the statistical advantage of placebo trials requiring fewer relapse events may be nullified by the higher dropout rates associated with these studies.

For patients requiring maintenance treatment, exposure to placebos is associated with increased risk of relapse and consequently a risk of undue harm. Inclusion of a placebo arm conflicts with the principle of clinical equipoise (when there is uncertainty whether a treatment will be beneficial), which requires the use of best available treatment as the control in a randomised trial. It also conflicts with the principle of beneficence, which requires that physicians should act in the best interest of each patient. Physicians often experience conflict of interests when participating in clinical trials, having to balance the interests of patients, academic reward, and, in the case of industry sponsored studies, financial incentives. Though Miller and Brody argued that clinical equipoise ignores the distinction between clinical trials and treatment, and that placebo controls are ethically justifiable in some situations, they mention placebo controlled trials in schizophrenia as an example where it would be difficult to justify the risks of symptom exacerbation for those randomised to placebos. ${ }^{15}$

There are also misgivings from a patient perspective, particularly around consent. In clinical settings it is difficult for people with schizophrenia to agree to take antipsychotics because of factors such as society's prejudice about psychiatric medication, deleterious side effects, and the person not having a strong enough bond of trust with their prescriber. The use of placebos in clinical trials runs the risk of fracturing trust, which is the cornerstone of the therapeutic relationship, with serious implications for ongoing treatment.

In addition to the ethical concerns, scientific considerations diminish the power of placebo controlled trials to establish efficacy. There is a risk of selection bias, both of patients and of investigators. Patients who are sceptical about the long term use of antipsychotics are more likely to agree to participate-indeed, fear of relapse was cited as one of the main reasons for patients not participating in a placebo controlled study. ${ }^{17}$ Also, many investigators refuse to participate in such trials, citing ethical concerns as the reasons. ${ }^{18} \mathrm{~A}$ further concern is that the high dropout rates in clinical trials using placebo $\operatorname{controls}^{19}$ reduce their statistical power. Also, there is a risk of unblinding as patients receiving placebos may sense that they are no longer taking an active medication. Finally, there is evidence, albeit weak, that sudden discontinuation of active treatment might provoke a psychosis in some patients, over and above the risk from the underlying illness. ${ }^{2}$ More research is required, including trials incorporating gradual and careful 
withdrawal of medication or further exploration of a low dose option, where clinical equipoise exists and where contingencies are arranged to minimise any harms from relapse.

We believe that a distinction needs to be drawn between short term efficacy and relapse prevention trials. Concerns regarding the use of placebos are greatest in relapse prevention trials.

Other study designs such as the use of an active control with a non-inferiority design represent a reasonable alternative to placebo controlled trials. ${ }^{80}$ A precedent is already established, as several widely used antipsychotics are registered for maintenance treatment for schizophrenia without placebo controlled relapse prevention studies having been conducted, most notably risperidone (both oral and long acting injectable), amisulpride, and olanzapine pamoate. ${ }^{7}$ Most placebo controlled relapse prevention trials have shown efficacy for the tested drug. For new drugs with a similar mechanism of action to those already approved it is therefore sufficient to rely on the short term efficacy results, the data on existing drugs, and maintenance treatment design strategies that do not use placebos.

\section{No evidence of harm or evidence of no harm}

The literature investigating the consequences of withholding treatment and the consequences of relapse in schizophrenia is inadequate. ${ }^{7}$ There are few well designed longitudinal studies assessing the psychosocial and biological consequences of exposure to placebos or relapse. Without such studies it cannot be assumed that patients experiencing relapses are not at risk of severe or lasting harm. No evidence of harm is different from evidence of no harm, ${ }^{21}$ and the burden must be to show no harm. There is cause for concern that a real risk exists.

Contributors and sources: The authors are clinicians with extensive experience in clinical trials in schizophrenia and a representative from a mental health advocacy group. RE, WWF, and NRS contributed to the overall conception of the article. RE wrote the first draft. All of the authors participated in telephone discussions, contributed to drafting the manuscript, and authors approved the final draft. The views expressed are those of the authors. RE is the guarantor.

Competing interests: We have read and understood BMJ policy on declaration of interests and declare the following. RE has participated in speakers/advisory boards and received honorariums from AstraZeneca, Bristol-Myers Squibb, Janssen, Lilly, Lundbeck, Servier, and Otsuka. He has received research funding from Janssen, Lundbeck, and AstraZeneca; WWF has received research grants from Otsuka, Janssen Cilag, and Lundbeck; advisory board honorariums from Lundbeck, Roche, Otsuka, Janssen Cilag, Takeda, Amgen, Teva, and Targacept; and speaker honorariums from Lundbeck, Janssen Cilag, Otsuka, Roche, and Takeda. He has stocks in MedAvante; SG received honorariums for lectures or advisory boards from Janssen-Cilag, Hoffmann-La Roche, Angelini ACRAF, and Lundbeck. JPM reports personal fees from Lilly, grants and personal fees from Merck, grants and personal fees from Sunovion, personal fees from Otsuka, personal fees from Alkermes, grants and personal fees from
Rovhe/Genentech, personal fees from Envivo, grants from GlaxoSmithKleine, and grants from Psychogenetics; NRS has received grant/research support from Otsuka, Neurocrine, and Genentech and has served as advisory board member/speaker for Roche, Forum, Sunovion, and Lundbeck.

Provenance and peer review: Not commissioned; externally peer reviewed.

1 Emsley R, Chiliza B, Asmal L. The evidence for illness progression after relapse in schizophrenia. Schizophr Res 2013;148:117-21. doi:10.1016/j.schres.2013.05.016 pmid: 23756298.

2 Moncrieff J. Antipsychotic maintenance treatment: time to rethink?PLoS Med 2015;12:e1001861. doi:10.1371/journal.pmed.1001861 pmid:26241954.

3 Wunderink L, Nieboer RM, Wiersma D, Sytema S, Nienhuis FJ. Recovery in remitted first-episode psychosis at 7 years of follow-up of an early dose reduction/discontinuation or maintenance treatment strategy: long-term follow-up of a 2-year randomized clinical trial. JAMA Psychiatry 2013;70:913-20. doi:10.1001/jamapsychiatry.2013.19 pmid: 23824214.

4 Zipursky RB, Menezes NM, Streiner DL. Risk of symptom recurrence with medication discontinuation in first-episode psychosis: a systematic review. Schizophr Res 2014;152:408-14. doi:10.1016/j.schres.2013.08.001 pmid:23972821.

5 Norman RM, Malla AK. Prodromal symptoms of relapse in schizophrenia: a review. Schizophr Bull 1995;21:527-39. doi:10.1093/schbul/21.4.527 pmid:8749881.

6 Leucht S, Tardy M, Komossa K. Heres S, Kissling W, Davis JM. Maintenance treatment with antipsychotic drugs for schizophrenia. Cochrane Database Syst Rev 2012;5:CD008016. doi:10.1002/14651858.CD008016.pub2. pmid:22592725.

7 Emsley R, Fleischhacker WW. Is the ongoing use of placebo in relapse-prevention clinical trials in schizophrenia justified? Schizophr Res 2013;150:427-33. doi:10.1016/j.schres. 2013.09.008 pmid:24094881.

8 Weijer C. Placebo-controlled trials in schizophrenia: are they ethical? Are they necessary? Schizophr Res 1999;35:211-8, discussion 227-36. doi:10.1016/S0920-9964( 98)00127-3 pmid:10093865.

9 Kane JM, Kishimoto T, Correll CU. Non-adherence to medication in patients with psychotic disorders: epidemiology, contributing factors and management strategies. World Psychiatry 2013:12:216-26. doi:10.1002/wps.20060 pmid:24096780.

10 Leucht S, Hierl S, Kissling W, Dold M, Davis JM. Putting the efficacy of psychiatric and general medicine medication into perspective: review of meta-analyses. Br J Psychiatry 2012;200:97-106. doi:10.1192/bjp.bp.111.096594 pmid:22297588.

11 World Medical Association. Declaration of Helsinki: ethical principles for medical research involving human subjects. JAMA 2013;310:2191-4. doi:10.1001/jama.2013.281053 pmid: 24141714.

12 International Conference on Harmonisation. Guidance E10: choice of control group and related issues in clinical trials. Step 4 version. $\mathrm{ICH}, 2000$.

13 European Medicines Agency Committee for Medicinal Products for Human Use. Guideline on clinical investigation of medicinal products, including depot preparations in the treatment of schizophrenia. EMA/CHMP/40072/2010. 9-20-2012.

14 US Food and Drug Administration Center for Drug Evaluation and Research. Guidance for industry: enrichment strategies for clinical trials to support approval of human drugs and biological products. Draft guidance. 2012. www.fda.gov/downloads/Drugs/.../ Guidances/UCM332181

15 Miller FG, Brody $\mathrm{H}$. What makes placebo-controlled trials unethical? Am J Bioeth 2002;2:3-9. doi:10.1162/152651602317533523 pmid:12189059.

16 Beasley CM Jr, , Sutton VK, Hamilton SH, et al. Olanzapine Relapse Prevention Study Group. A double-blind, randomized, placebo-controlled trial of olanzapine in the prevention of psychotic relapse. J Clin Psychopharmacol 2003;23:582-94. doi:10.1097/01.jcp. 0000095348.32154.ec pmid:14624189.

17 Hummer M, Holzmeister R, Kemmler G, et al. Attitudes of patients with schizophrenia toward placebo-controlled clinical trials. J Clin Psychiatry 2003;64:277-81. doi:10.4088/ JCP.v64n0308 pmid:12716268.

18 Fleischhacker WW, Burns T. European Group for Research in Schizophrenia. Feasibility of placebo-controlled clinical trials of antipsychotic compounds in Europe. Psychopharmacology (Berl) 2002;162:82-4. doi:10.1007/s00213-002-1060-z pmid: 12107622.

19 Kemmler G, Hummer M, Widschwendter C, Fleischhacker WW. Dropout rates in placebo-controlled and active-control clinical trials of antipsychotic drugs: a meta-analysis. Arch Gen Psychiatry 2005:62:1305-12. doi:10.1001/archpsyc.62.12.1305 pmid:16330718.

20 Fleischhacker WW, Czobor P, Hummer M, Kemmler G, Kohnen R, Volavka J. Placebo or active control trials of antipsychotic drugs? Arch Gen Psychiatry 2003;60:458-64. doi: 10.1001/archpsyc.60.5.458 pmid:12742866.

21 Kim SY. Benefits and burdens of placebos in psychiatric research. Psychopharmacology (Berl) 2003;171:13-8. doi:10.1007/s00213-003-1458-2 pmid:12684741.

Published by the BMJ Publishing Group Limited. For permission to use (where not already granted under a licence) please go to http://group.bmj.com/group/rights-licensing/ permissions 


\section{Summary points}

Concerns regarding the use of placebos in schizophrenia are greatest in relapse prevention trials Measures to identify early signs of relapse are not always effective, and rescue interventions may not always be able to prevent recurrence Research is limited but there is sufficient cause for concern that withdrawing treatment risks undue patient harm Non-inferiority comparative study designs represent a reasonable alternative to placebo controlled trials 